\title{
Correction: Existing Mobile Phone Apps for Self-Care Management of People With Alzheimer Disease and Related Dementias: Systematic Analysis
}

Yuqi Guo ${ }^{1}, \mathrm{MSW}, \mathrm{PhD}$; Fan Yang ${ }^{2}, \mathrm{MSW}, \mathrm{PhD} ; \mathrm{Fei} \mathrm{Hu}{ }^{3}, \mathrm{PhD}$; Wei $\mathrm{Li}^{4}, \mathrm{MD}, \mathrm{PhD}$; Nicole Ruggiano ${ }^{5}, \mathrm{PhD}$; Hee Yun Lee ${ }^{5}, \mathrm{PhD}$

\footnotetext{
${ }^{1}$ School of Social Work, University of North Carolina at Charlotte, Charlotte, NC, United States

${ }^{2}$ Social Welfare Program, School of Public Administration, Dongbei University of Finance and Economics, Dalian, China

${ }^{3}$ College of Engineering, University of Alabama, Tuscaloosa, AL, United States

${ }^{4}$ School of Health Professions, University of Alabama at Birmingham, Birmingham, AL, United States

${ }^{5}$ School of Social Work, University of Alabama, Tuscaloosa, AL, United States
}

\section{Corresponding Author:}

Fan Yang, MSW, PhD

Social Welfare Program

School of Public Administration

Dongbei University of Finance and Economics

219 Quanxue Hall

217 Jianshan Street, Shaohekou District

Dalian, 116025

China

Phone: 8641184710562

Fax: 8641184710562

Email: fyang10@dufe.edu.cn

\section{Related Article:}

Correction of: https://aging.jmir.org/2020/1/e15290/

(JMIR Aging 2020;3(1):e18754) doi: 10.2196/18754

The authors of "Existing Mobile Phone Apps for Self-Care Management of People With Alzheimer Disease and Related Dementias: Systematic Analysis" (JMIR Aging 2020;3(1):e15290) noticed several errors in the author information of their published article.

Author Fei Hu's academic degree information has been corrected from "MD" to " $\mathrm{PhD}$ ".

Author Wei Li's academic degree information has been corrected from "MD" to "MD, PhD". Furthermore, the affiliation listed for author Wei Li has been revised from:
College of Engineering, University of Alabama,

Tuscaloosa, AL, United States

to the following:

School of Health Professions, University of Alabama at Birmingham, Birmingham, AL, United States

The correction will appear in the online version of the paper on the JMIR website on May 19, together with the publication of this correction notice. Because this was made after submission to PubMed, PubMed Central, and other full-text repositories, the corrected article has also been resubmitted to those repositories.

This is a non-peer-reviewed article. Submitted 17.03.20; accepted 17.03.20; published 19.05.20.

Please cite as:

Guo Y, Yang F, Hu F, Li W, Ruggiano N, Lee HY

Correction: Existing Mobile Phone Apps for Self-Care Management of People With Alzheimer Disease and Related Dementias: Systematic Analysis

URL: http://aging.jmir.org/2020/1/e18754/

doi: $\underline{10.2196 / 18754}$

PMID: 32427566 
(C) Yuqi Guo, Fan Yang, Fei Hu, Wei Li, Nicole Ruggiano, Hee Yun Lee. Originally published in JMIR Aging (http://aging.jmir.org), 19.05.2020. This is an open-access article distributed under the terms of the Creative Commons Attribution License (https://creativecommons.org/licenses/by/4.0/), which permits unrestricted use, distribution, and reproduction in any medium, provided the original work, first published in JMIR Aging, is properly cited. The complete bibliographic information, a link to the original publication on http://aging.jmir.org, as well as this copyright and license information must be included. 